\title{
Ruth McKernan
}

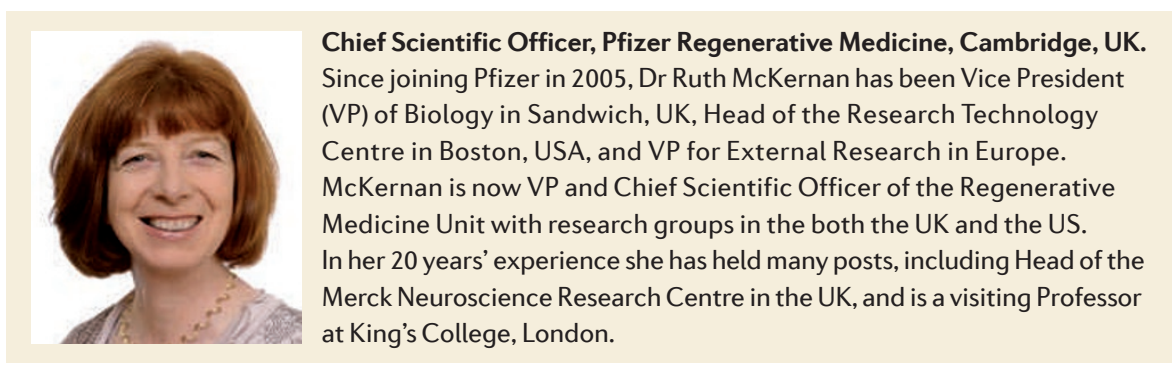

Prior to the creation of Pfizer Regenerative Medicine, how was Pfizer using

stem-cell-based technologies?

Pfizer has been using stem cells for at least 12 years as tools in drug discovery. For example, we screened our entire chemical collection - more than 2 million compounds - on neurons differentiated from mouse embryonic stem cells (ESCs) because the receptor that we were interested in was not available in other cell systems. We have also done small interfering RNA screens and small-molecule screens on bone marrow cells differentiated into an adult stem cell type, so it's certainly an area of science that we have been comfortable with for more than a decade.

What were the main reasons behind the decision to create Pfizer Regenerative Medicine?

One reason was that it was a natural extension of the work that we were already doing. For example, in order to screen 2 million compounds we had to rigorously produce consistent cells. We can apply our learning from that to cell-based therapies. Also, the timing seemed right with regard to the speed at which the science is moving, the creation of induced pluripotent stem (iPS) cells and the increasing number of clinical studies underway. In addition, there was recognition in Pfizer that we wanted to be much more innovative, as exemplified by the creation of the Biotherapeutics and Bioinnovation Centre and the change in philosophy to pursue and partner with the best external science. All of these things aligned when a small group of us proposed that we move into regenerative medicine, to research stem cells as therapeutics rather than tools. I found a lot of support and a lot of confidence in the company about the potential of stem cells. They offer the next evolution of therapeutics and may provide a more holistic benefit when single molecules aren't enough and biologics have run their course. After all, they are modular factories secreting a physiological combination of paracrine factors, honed by evolution and therefore less likely to produce unexpected toxicities. This new research unit represents an exciting opportunity for Pfizer to be at the forefront of this emerging area of science.

What are the main challenges for companies developing stem-cell-based therapies, and how is Pfizer addressing them? Stem-cell-based therapies cover a very large spectrum and I would include small molecules or biologics that modify cell behaviour or cell fate, adult stem-cell-based therapies that don't integrate into tissues and ESCs that differentiate into target cells and could integrate into tissues. We have a lot more confidence and experience in small molecules and biologics that modify cell behaviour or cell fate. But, when it comes to stem cells, one has to consider how to expand them, how to control them and how to be confident of the cells' purity - to ensure they are not contaminated with other cell types. How cells are controlled in vitro before their application in vivo is a significant challenge and not to be underestimated. We are partnering with experienced academics and biotechs to address these challenges.

Following the first FDA approval of a clinical trial for an embryonic stem-cell-based therapy (from Geron), what do you think are the key regulatory issues for such products? There are many areas where we can work with the regulators to understand how cells behave when they are put into the body. We need to understand the full spectrum of where the cells can go, what they can become, how their fate is determined and the degree of contamination by other cell types - in essence: safety, efficacy, purity and potency. For example, administering a pure population of pluripotent cells, if they can become many different cell types, could be a much bigger challenge than giving a mixed population that generates one cell type only. There are going to be significant challenges if we are using cells derived from human ESCs, and further issues if we were to consider cells derived from iPS cells. I think there could be fewer hurdles for adult stem cells than those that have been modified or expanded. In theory, the further you move from the pluripotent state, the less likely it is that there should be problems, although we don't know that for certain yet.

How is Pfizer engaging with the academic and biotech communities that have expertise in stem cell R\&D?

I'm spending most of my time at the moment talking to academic groups and biotech companies about their research and potential areas of collaboration. We are a very externally focused research unit and I do not want to reinvent something that has already been done; there is so much research and development going on in this space. We have already put together partnerships with several biotech companies and university academic groups to collaborate across the spectrum of adult cells, embryonic cells and iPS cells. One partnership that we announced in December 2008 is with Novocell [a stem cell engineering company dedicated to creating, delivering and commercializing cell and drug therapies to treat diabetes] where we will be working with them to understand how to control the production of $\beta$-cells from ESCs. We will be using our small molecules and Novocell's cell expertise to identify molecules that are important in that pathway. It's a matter of bringing together what pharma does very well - developing small molecules and biologics - with the biotech companies' expertise in understanding cell biology and how to control and manage stem cells. So I think it could be a great marriage. 